\title{
Carbon capture in living aerial biomass in Tingo María National Park
}

\section{Captura de carbono en biomasa aérea viva en el Parque Nacional Tingo María}

\author{
Luis Eduardo Ore Cierto ${ }^{l}$, Julio Brayan Vargas Arata $^{l}$, Wendy Caroline Loarte Aliaga ${ }^{l}$, Juan Daniel \\ Oré Cierto ${ }^{l}$ and Edilberto Díaz Quintana ${ }^{l}$
}

${ }^{1}$ Universidad Nacional Agraria de la Selva, Perú.

\begin{abstract}
The present research work, has the objective of determining the quantity of carbon in the live aerial arboreal biomass; to do so, seven $50 \mathrm{~m} \times 50 \mathrm{~m}$ plots were defined; randomly distributed within the area of the current research; twenty eight families and seventy three arboreal species were identified from a total of 1837 specimens; the family with the most abundance was Rubiaceae, the specie with the most abundance was Cinchona pubescens Vahl (Quina amarillo) with 143 specimens identified. The quantity of stored carbon in the aerial arboreal biomass was an average of $70.08 \mathrm{tC} / \mathrm{ac}$ with respect to the plots studied; statistically, the stored carbon has a heterogenous behavior within the area in study; due to the fact that it is found to be influenced by the height and type of vegetation category; at 1066 - 1187 masl the greatest quantity of carbon was found; and the mature trees influence the quantity of carbon at a $50 \%$, followed by the rest of the categories.
\end{abstract}

Keywords: carbon, live aerial biomass, absolute abundance, wilderness.

\section{RESUMEN}

El presente trabajo de investigación tiene como objetivo determinar el carbono en la biomasa aérea arbórea viva; por lo que se delimitaron 7 parcelas de $50 \mathrm{~m}$ x 50m; distribuidos aleatoriamente en toda el área de la presente investigación; se identificaron 28 familias, 73 especies arbóreas de un total de 1837 individuos en total; la familia de mayor abundancia fue la Rubiaceae, la especie de mayor abundancia absoluta fue la Cinchona pubescens Vahl (Quina amarilla) con 143 individuos identificados. El promedio de carbono en la biomasa aérea arbórea viva fue de $70.08 \mathrm{tC} / \mathrm{ha}$ con respecto a las parcelas de estudio; estadísticamente el carbono almacenado tiene un comportamiento heterogéneo dentro del área de estudio; debido a que se encuentra influenciada por la altitud y categoría de tipo de vegetación; a 1066 - 1187 msnm se halló la mayor cantidad de carbono; y los árboles maduros, influencia un 50\% en la cantidad de carbono, seguido del resto de categorías.

Palabras clave: carbono, biomasa aérea viva, abundancia absoluta, zona silvestre.

Luis Eduardo Ore Cierto. ORCID: https://orcid.org/0000-0003-2836-2436 email: luisore21793@ gmail.com Julio Brayan Vargas Arata. ORCID: https://orcid.org/0000-0002-7592-4000 email: jbva.1226@gmail.com Wendy Caroline Loarte Aliaga. ORCID: https://orcid.org/0000-0001-9489-046X email: wendy_loarte_aliaga@outlook.com.pe Juan Daniel Oré Cierto. ORCID: https://orcid.org/0000-0002-1788-5146 email: jore2028@ gmail.com

Edilberto Díaz Quintana. ORCID: https://orcid.org/0000-0001-7498-109X email: edilberto.diaz@unas.edu.pe 


\section{INTRODUCCIÓN}

$\mathrm{T}$ he Intergovernmental Panel on Climate Change (IPCC) (2018) mentions that there is a growing global concern about the increase in the concentration of carbon dioxide $(\mathrm{CO} 2)$ in the earth's atmosphere, which is considered one of the six main gases involved in the greenhouse effect ( GHG); and that contribute in greater proportion to climate change. One way to reduce $\mathrm{CO} 2$ emissions is to capture it, fix it and store it for as long as possible in plant biomass. The main problem, which encourages the realization of the investigation is the increase in temperature within our locality. According to the data obtained from the José Abelardo Quiñones meteorological station (Tingo María), they indicate that from 1940 to 1970 the minimum temperature has increased by approximately $1{ }^{\circ} \mathrm{C}$, likewise until 2005 it had increased by $1.1^{\circ} \mathrm{C}$, for the year 2018 the temperature increased $0.8^{\circ} \mathrm{C}$, so probably the increase in different economic activities and population growth are generating a large amount of $\mathrm{CO} 2$, for which the temperature is increasing, because these parameters have a relationship directly proportional. The IPCC indicates that from the $1990 \mathrm{~s}$ to the present the temperature has been increasing due to the large generation of greenhouse gases due to industrialization, it also reports that the change in temperature greater than and/or equal to $2{ }^{\circ} \mathrm{C}$ generates large impacts on natural and human systems (IPCC, 2018); Therefore, it recommends maintaining a temperature change of less than $1.5{ }^{\circ} \mathrm{C}$ since the world will suffer fewer negative impacts in the intensity and frequency of extreme events, in resources, ecosystems,

The objective of this research work is to estimate carbon sequestration in living tree aerial biomass in the wild area of the Tingo María National Parke.

\section{Materials AND MethodS}

\section{Location and description of the study area}

The study was carried out in the forest area of the Tres de Mayo - Rio Oro section in the wild zone of the Tingo María National Park (PNTM). Its limits are: To the North: with the Caserío de Bella. To the East: with the Tingo María National Park. To the South: with the populated center of Tambillo Chico - Grande. To the West: with the tourist area of the Tingo María National Park.

\section{Materials and equipment}

El trabajo de investiga For the development of the different field tasks, the following materials were used: $2 \mathrm{~m}$ tape measure, $50 \mathrm{~m}$ winch, field notebook, manila envelopes, machete, mica plates, raffia, $12^{\prime \prime}$ head nails and indelible markers Equipment such as: GARMIN 62s GPS, 16x SONY digital camera, BRUNTON clinometer, BRUNTON compass, HENKEL 03 decimal precision scale, MEMMERT stove, ASUS N56J laptop, and software such as: Microsoft Word, Microsoft Excel, QGIS 10.2.1 and IBM SPSS Statistics Trial (Classic Student).

\section{Methodology}

Determination of the absolute abundance of tree species identified in the forests of the Tres de Mayo - Rio Oro stretch in the wild area of the PNTM

\section{Determination of sample size}

A simple random sampling was carried out, for which the error was $15 \%$ with a confidence coefficient of $85 \%$, with these data the sample size was determined. Following the standard statistical sampling procedures, the plot size was 0.25 hectares $(50 \mathrm{mX} 50 \mathrm{~m})$, the following formula was used (Morillas, 2007).

$$
\mathrm{n}=\frac{\mathrm{N} * \mathrm{z}_{1-\alpha / 2}^{2} * \sigma^{2}}{(\mathrm{~N}-1) * \mathrm{E}^{2}+\mathrm{z}_{1-\alpha / 2}^{2} * \sigma^{2}}
$$

Where:

$\mathrm{n} \quad=$ Number of plots of size $0.25(\mathrm{Ha})$

$\mathrm{N} \quad=$ Total number of study plots $(\mathrm{Ha})($ value $=3516.4)$

$\mathrm{z} 1-\alpha / 2=$ Value of $\mathrm{Z}$ according to confidence level (value $=$ $1.44)$

$\sigma \quad=$ Variance $($ value $=0.28)$

AND $=$ Error $($ value $=15 \%)$

\section{Delimitation of plots}

According to Manta (1988), in each of the delimited plots sub-plots were made for the sampling of low poles ( 2 of $10 \mathrm{x}$ $10 \mathrm{~m}$ ), tall poles ( 2 of $25 \times 25 \mathrm{~m}$ ), poles and mature trees in total of the plot $(1$ of $50 \times 50 \mathrm{~m})$.

\section{Tree inventory}

Table 01.

The categories evaluated in the trees

\begin{tabular}{cc}
\hline Category & Diameter \\
\hline low pole & $>1.3 \mathrm{~m}$ high at $\varnothing<5 \mathrm{~cm}$ \\
tall pole & $5 \mathrm{~cm}<\varnothing \leq 10 \mathrm{~cm}$ \\
stem & $10 \mathrm{~cm}<\varnothing \leq 40 \mathrm{~cm}$ \\
mature trees & $\varnothing>40 \mathrm{~cm}$ \\
\hline
\end{tabular}

Source: Blanket (1988)

\section{Tree coding and labeling}

Each tree species found was coded with mica plates, the coding started from number one until the end of the count, and the plate was placed approximately at the height where the diameter of the tree was measured, likewise the evaluated category was labeled and the sub plot to which it belongs: for low poles (B), for tall poles (L), for poles (F) and for mature trees (A); for this, the nails were used to hold the plates; For this work, the modified label model (Pinelo, 2000) was used. 
ISSNe: 2617-9156

\section{Species abundance calculation}

According to Lamprecht (1990), the absolute abundance (Aa) of a species is expressed by the total number of individuals "ni" of each species existing in the study area:

Where:

$$
\mathrm{Aa}=\sum \mathrm{ni}
$$

Aa $=$ Absolute abundance.

$\Sigma$ ni $=$ Sum of number of equal species.

Determination of biomass area and carbon stored in tree species in the section Tres de Mayo - Rio Oro in the wild area of the Tingo María National Park

\section{Tree diameter measurement}

The diameter of the trees was measured in the bark, at breast height $(1.3 \mathrm{~m})$, this diameter is called: diameter at breast height (dbh) (Ortiz, 1993), which was measured with a tape measure reinforced (so that the measurement is as accurate as possible).

\section{Tree height measurement}

Vallejo et al. (2005) indicates that in order to obtain the height of the trees, the estimation must be made from the ground to the top of the crown of each individual.

For the direct estimation of the heights of the trees, the Blumme-Leiss method was used, it should be noted that when the height is estimated, not much precision is achieved, and an approximate error of up to $1 \mathrm{~m}$ can be obtained; To determine the error in the height estimation, a control measurement was made with a clinometer to a sample of trees as learning and reduction of the error in the estimation (Domínguez, 2010).

\section{Sample extraction}

For each species of tree found within the plot, a sample of wood was collected from the stem of the tree; these samples were cylindrical which allowed studying the humidity and basic density of the forest species, this estimation method is used more frequently for such studies (Borrero, 2012).

\section{Dry weight calculation}

A sample of each tree species found was extracted, which was then stored in an official manila envelope to later be dried in an oven at $75^{\circ} \mathrm{C}$ in the laboratory until the constant dry weight was found and weighed on the scale. precision (Wieman and Williamson, 1989).

\section{Basic Density Calculation}

Murray and Jacobson (1982), for the determination of the basic density of the wood, the wood samples were used and it was determined by dividing the green volume submerged in water by the dry weight of the wood.

\section{Calculation of aerial tree biomass}

To determine the aerial biomass, an exponential equation established by Chave et al. (2005) referenced below:

$$
\begin{aligned}
& \mathrm{BT}=\exp (-2.977 \\
& \left.+\operatorname{Ln}\left(\delta * \mathrm{Dap}^{2} * \mathrm{~h}\right)\right)
\end{aligned}
$$

Where:

$$
\begin{array}{ll}
\mathrm{BT} & =\text { Aerial biomass }(\mathrm{Kg}) \\
\mathrm{Ln} & =\text { natural logarithm } \\
\mathrm{dbh} & =\text { Diameter at breast height or DBH }(\mathrm{cm}) \\
\mathrm{h} & =\text { Total tree height }(\mathrm{m}) \\
\delta & =\text { Basic density of wood }(\mathrm{g} / \mathrm{cm} 3) \\
2,977 & =\text { Constant }
\end{array}
$$

\section{Calculation of stored carbon}

After obtaining the biomass, the stored carbon was calculated with the following formula proposed by IPPC (2003):

$$
\mathrm{CA}=\mathrm{BT} * 0.5
$$

Where:

AC = Carbon stored ( $\mathrm{t} \mathrm{C/ha)}$

BT $=$ Biomass $(\mathrm{t} / \mathrm{ha})$

\begin{tabular}{|c|c|c|c|c|}
\hline $\begin{array}{l}\text { Variation } \\
\text { Source }\end{array}$ & $\begin{array}{c}\text { Degrees of } \\
\text { freedom }\end{array}$ & Sum of squares & Middle square & F-value \\
\hline Regression & $\mathrm{k}$ & $\mathrm{SCR}=\sum\left(\widehat{\mathrm{Y}}_{\mathrm{i}}-\overline{\mathrm{Y}}\right)^{2}$ & $\mathrm{CMR}=\frac{\mathrm{SCR}}{\mathrm{k}}$ & $\mathrm{F}=\frac{\mathrm{CMR}}{\mathrm{CME}}$ \\
\hline Error & $\mathrm{n}-\mathrm{k}-1$ & $\mathrm{SCE}=\sum\left(\mathrm{Y}_{\mathrm{i}}-\widehat{\mathrm{Y}}\right)^{2}$ & $\mathrm{CME}=\frac{\mathrm{SCE}}{\mathrm{n}-\mathrm{k}-1}$ & \\
\hline Total & $n-1$ & $\mathrm{SCT}=\sum\left(\mathrm{Y}_{\mathrm{i}}-\overline{\mathrm{Y}}\right)^{2}$ & & \\
\hline
\end{tabular}

$0.5=$ Conventional constant indicated by the IPCC (Carbon).

Determination of the behavior of stored carbon based on altitude and category of vegetation type

Statistical analysis was performed with the help of the IBM SPSS Statistics Trial (Classic student) software, and Microsoft Excel:

Table 02.

Analysis of variance (ANVA)

Source: Allen (2001) 


\section{Results}

Determination of the absolute abundance of the tree species identified in the forests of the Tres de Mayo - Rio Oro section in the wild area of the Tingo María National Park (PNTM)

Table 03.

Absolute abundance of tree species by evaluated plots.

\begin{tabular}{|c|c|c|c|c|c|c|c|c|c|c|}
\hline Family & Scientific name & Common name & $\mathrm{P} 1$ & $\mathrm{P} 2$ & P3 & $\mathrm{P} 4$ & P5 & P6 & $\mathrm{P} 7$ & oh \\
\hline \multirow{3}{*}{ Annonaceae } & fusaea peruviana RE Fries & anonilla & 0 & 0 & two & 0 & 3 & 5 & 5 & $\begin{array}{c}\text { fifte } \\
\text { en }\end{array}$ \\
\hline & Guatteria hyposericeadiels & Carahuasca & 0 & 0 & 3 & 0 & 0 & 0 & two & 5 \\
\hline & annona scandens aff. diels & Yanavarilla & 17 & 19 & 0 & 0 & 6 & 5 & 24 & 71 \\
\hline \multirow{2}{*}{ Anacardiaceae } & Spondias mombin L. & ubos & 0 & 0 & 0 & 0 & 0 & 0 & 5 & 5 \\
\hline & Mangifera indica $\mathrm{L}$. & Mango & 0 & 4 & 0 & 0 & 0 & 0 & 0 & 4 \\
\hline \multirow[t]{2}{*}{ Apocynaceae } & $\begin{array}{c}\text { Rhigospira quadrangularis (Muell. } \\
\text { Arg.) Miers }\end{array}$ & Guayavilla & two & 4 & $\begin{array}{c}\text { fifte } \\
\text { en }\end{array}$ & $\begin{array}{c}\text { tw } \\
\mathrm{o}\end{array}$ & 23 & 17 & 0 & 63 \\
\hline & Aspidosperma excelsum aublet & caspi rowing & 0 & 0 & 6 & 0 & 4 & one & 5 & 16 \\
\hline \multirow[t]{2}{*}{ arecaceae } & Socratea exorrhiza H. Moore & Pona & 0 & 0 & 31 & 6 & 24 & $\begin{array}{l}\text { twe } \\
\text { nty }\end{array}$ & 0 & 81 \\
\hline & maximiliana maripa (Aubl) Drude & Maximilian & 0 & 0 & 0 & 10 & 0 & 0 & 0 & 10 \\
\hline \multirow{2}{*}{ Burseraceae } & $\begin{array}{c}\text { Protium decandrum (Aublet) } \\
\text { Marchand }\end{array}$ & Copal & 0 & 0 & 3 & 0 & 9 & 6 & 0 & 18 \\
\hline & bursera graveolens HBK & Lignum vitae & 7 & 0 & 0 & 0 & 3 & 0 & 25 & 35 \\
\hline Bixaceae & bixa orellana $\mathrm{L}$. & achiote caspi & 0 & 0 & 0 & 0 & 0 & 0 & 3 & 3 \\
\hline Bignoniaceae & Copaia Jacaranda (Aubl.) D. Don & Huamansamana & 0 & 0 & 7 & 0 & 0 & 0 & 0 & 7 \\
\hline Caricaceae & Jacaratia digitata(Poepp.) Solms & papaya caspi & 0 & 0 & 0 & 0 & 0 & 0 & 5 & 5 \\
\hline Caryocaraceae & Anthodiscus gutierrezii L. Wms. & jacket & 0 & two & 0 & 0 & 0 & 0 & 0 & two \\
\hline Clusiaceae & Garcinia macrophylla Mart & chickadee & 0 & 0 & 0 & 0 & 6 & 5 & 0 & $\begin{array}{l}\text { elev } \\
\text { en }\end{array}$ \\
\hline \multirow{3}{*}{ Combretaceae } & $\begin{array}{l}\text { Terminalia oblongata (Ruiz \& Pav.) } \\
\text { Steud. }\end{array}$ & raffle & 0 & 0 & $\begin{array}{c}\text { elev } \\
\text { en }\end{array}$ & 0 & 25 & $\begin{array}{l}\text { fift } \\
\text { een }\end{array}$ & 0 & 51 \\
\hline & $\begin{array}{c}\text { Amazonian Terminalia (JF Gmelin) } \\
\text { Exell. }\end{array}$ & yacushapana & 5 & 25 & 0 & 0 & 14 & 10 & two & 56 \\
\hline & Terminalia catappa (L.) Lour. & Almond & one & 0 & one & 0 & 6 & 6 & 3 & 17 \\
\hline \multirow{8}{*}{ Fabaceae } & Schizolobium amazonicum huber & Pashaco & 0 & 0 & 0 & 4 & 0 & 0 & 0 & 4 \\
\hline & towering inga Mark & Shimbillo & 8 & 16 & 23 & 9 & 14 & 9 & two & 81 \\
\hline & Sclerolobium melinonii harms & ucshaquiro white & 0 & 0 & $\begin{array}{c}\text { elev } \\
\text { en }\end{array}$ & 0 & 3 & two & 0 & 16 \\
\hline & Bauhinia guianensis aublet & ox leg & 0 & 0 & 0 & 0 & 0 & 0 & two & two \\
\hline & Apuleia molaris Spruce ex Bentham & caspi cabbage & 0 & 0 & 0 & 0 & two & 0 & 0 & two \\
\hline & Cassia reticulata Wilid. & Broom & 0 & 0 & 0 & 0 & one & two & 4 & 7 \\
\hline & $\begin{array}{c}\text { Balizia pedicellaris(DC.) Barneby \& } \\
\text { JW Grimes }\end{array}$ & pashaco vilco & two & 0 & 0 & 0 & 0 & 0 & one & 3 \\
\hline & Erythrina ulei harms & Amasisa & 0 & 0 & 0 & 18 & 0 & one & 4 & 23 \\
\hline \multirow{2}{*}{ Hypericaceae } & Vismia cayennensis(Jacquin) Person & Pichirina & 0 & two & 0 & 0 & one & 0 & 0 & 3 \\
\hline & Symphonia globulifera L.f. & sulfur stick & 0 & 0 & 0 & 0 & 10 & two & 0 & 12 \\
\hline \multirow[t]{3}{*}{ Lamiaceae } & Vitex psudolea Rusby & pali dog & 5 & 0 & 0 & 0 & 19 & 8 & 4 & 36 \\
\hline & Amazon Aniba Meiz & Moena & 0 & 0 & 3 & 0 & 0 & 0 & 0 & 3 \\
\hline & Aniba Cinnamon (HBK) Mez. & cinnamon moena & 0 & 0 & two & 0 & 0 & 0 & 0 & two \\
\hline \multirow[t]{4}{*}{ Lauraceae } & Aniba perutilis Hemsley Kew & black moena & 6 & 0 & $\begin{array}{l}\text { twe } \\
\text { nty- } \\
\text { one }\end{array}$ & 0 & $\begin{array}{c}\text { twe } \\
\text { nty } \\
- \\
\text { one }\end{array}$ & 10 & 8 & 66 \\
\hline & persea mollis (Kunth) Spreng. & moena avocado & 0 & 0 & one & 0 & 0 & 0 & 0 & one \\
\hline & $\begin{array}{l}\text { Mezilauros synandra } \\
\text { (Mez)Kosterm. }\end{array}$ & Moena & 0 & 0 & two & 0 & 0 & 0 & 0 & two \\
\hline & $\begin{array}{c}\text { Nectandra capanahuensis } \mathrm{O} . \\
\text { Schmidt }\end{array}$ & Yellow Moena & 0 & 0 & 9 & 0 & 9 & 6 & two & 26 \\
\hline
\end{tabular}


Revista de Investigación Científica Tayacaja Universidad Nacional Autónoma de Tayacaja Daniel Hernández Morillo, Perú Volumen 4 Número 2 - 2021

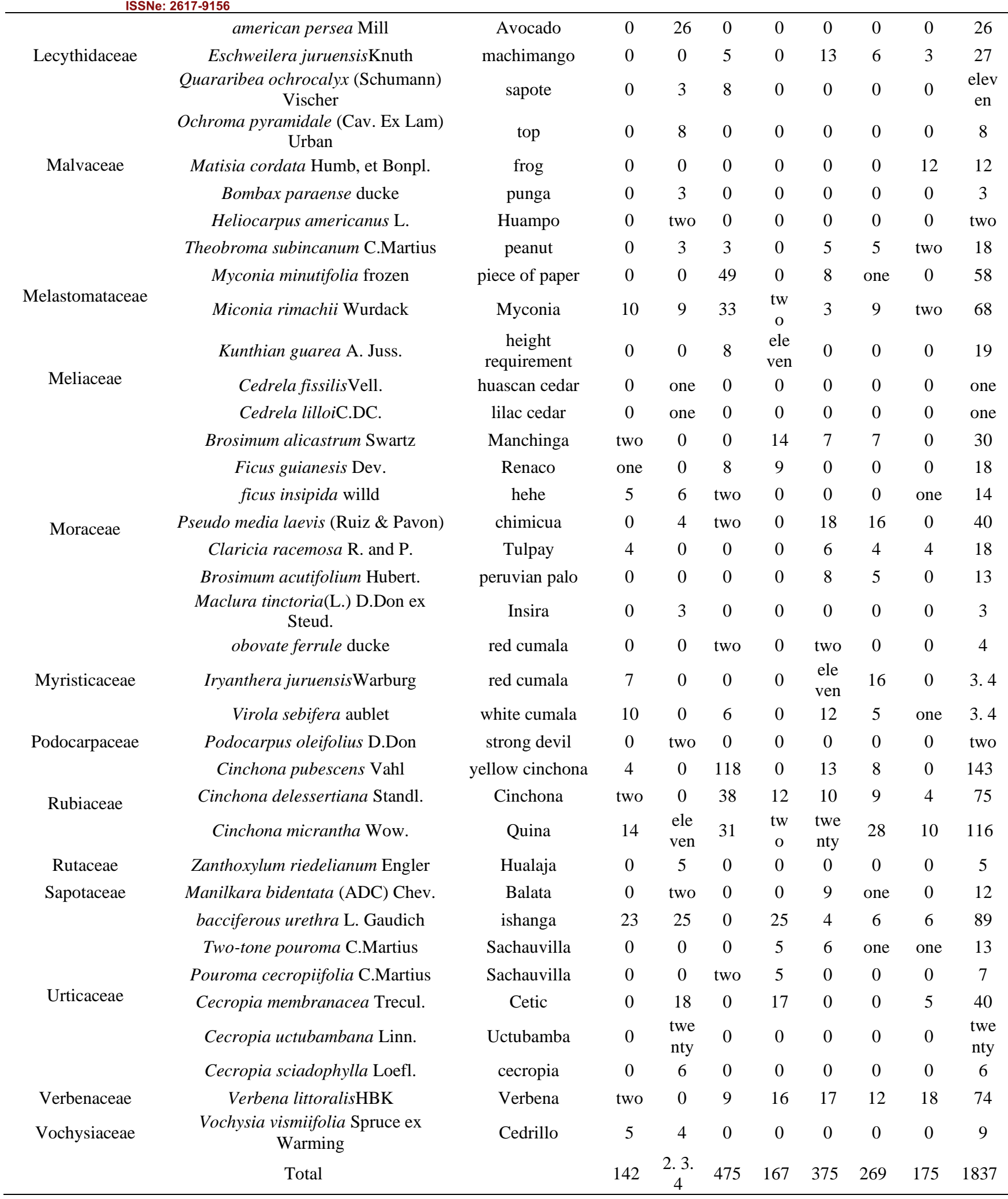

* P1, P2, P3, P4, P5, P6, P7: Number of plots

* Aa: Absolute abundance

Table 03 shows the absolute abundance of the species, likewise the total number of species identified were 1837 individuals as shown in the annexes, resulting in the inventory of 28 identified families, with a variety of 73 species in the 7 plots evaluated in the study. The most abundant family is Rubiaceae followed by the Urticaceae family. The species with the highest absolute abundance is the Cinchona pubescens Vahl (Quina amarillo) with 143 individuals, followed by the Cinchona micrantha Vahl. (Quina) with 116 individuals; the least abundant species was Persea mollis (Kunth) Spreng. (Palta moena), the species Cedrela lilloi C.DC. (Lilac cedar) and the species Cedrela fissilis Vell. (Cedro huasca) with 1 identified individual each. 
Determination of biomass area and carbon stored in tree species in the section Tres de Mayo - Rio Oro in the wild area of the Tingo María National Park (PNTM)

Table 04.

Basic density of tree species.

\begin{tabular}{|c|c|c|c|c|}
\hline Scientific name & Common name & $P(g)$ & $\mathrm{V}(\mathrm{cm} 3)$ & $\delta(\mathrm{g} / \mathrm{cm} 3)$ \\
\hline fusaea peruviana RE Fries & anonilla & 32.66 & 65 & 0.50 \\
\hline Guatteria hyposericeadiels & Carahuasca & 9.22 & $\begin{array}{l}\text { twenty- } \\
\text { one }\end{array}$ & 0.44 \\
\hline annona scandens aff. diels & Yanavarilla & 3.16 & 3 & 0.53 \\
\hline Spondias mombin L. & ubos & 9.3 & 26 & 0.36 \\
\hline Mangifera indica L. & Mango & 24.6 & 60 & 0.41 \\
\hline Rhigospira quadrangularis (Muell. Arg.) Miers & Guayavilla & 6.4 & 25 & 0.26 \\
\hline Aspidosperma excelsum aublet & caspi rowing & 28.71 & fifty & 0.57 \\
\hline Socratea exorrhiza H. Moore & Pona & 5.14 & twenty & 0.26 \\
\hline maximiliana maripa (Aubl) Drude & Maximilian & 2.06 & 6 & 0.34 \\
\hline Protium decandrum (Aublet) Marchand & Copal & 9.04 & 13 & 0.70 \\
\hline bursera graveolens HBK & Lignum vitae & 22.78 & 26 & 0.88 \\
\hline Ochroma pyramidale (Cav. Ex Lam) Urban & top & 7.1 & 38 & 0.19 \\
\hline Matisia cordata Humb, et Bonpl. & frog & 5.42 & 13 & 0.42 \\
\hline Bombax paraense ducke & punga & 12.49 & 31 & 0.40 \\
\hline bixa orellana $\mathrm{L}$. & achiote caspi & 10.8 & 26 & 0.42 \\
\hline Copaia Jacaranda (Aubl.) D. Don & Huamansamana & 11.1 & 28 & 0.40 \\
\hline Jacaratia digitata(Poepp.) Solms & papaya caspi & 16.32 & 41 & 0.40 \\
\hline Anthodiscus gutierrezii L. Wms. & jacket & 22.79 & 46 & 0.50 \\
\hline Two-tone pouroma C.Martius & Sachauvilla & 6.3 & 18 & 0.35 \\
\hline Pouroma cecropiifolia C.Martius & Sachauvilla & 40.85 & 118 & 0.35 \\
\hline Cecropia membranacea Trecul. & Cetic & 4.06 & 12 & 0.34 \\
\hline Cecropia uctubambana Linn. & Uctubamba & 76.39 & 105 & 0.73 \\
\hline Vismia cayennensis(Jacquin) Person & Pichirina & 7.13 & 14 & 0.51 \\
\hline Symphonia globulifera L.f. & sulfur stick & 29.58 & 57 & 0.52 \\
\hline Garcinia macrophylla Mart & chickadee & 5.78 & 19 & 0.30 \\
\hline Terminalia oblongata (Ruiz \& Pav.) Steud. & raffle & 28.12 & 48 & 0.59 \\
\hline Amazonian Terminalia (JF Gmelin) Exell. & yacushapana & 33.64 & 51 & 0.66 \\
\hline Terminalia catappa (L.) Lour. & Almond & 10.13 & twenty & 0.51 \\
\hline Schizolobium amazonicum huber & Pashaco & 11.45 & 29 & 0.39 \\
\hline towering inga Mark & Shimbillo & 15.89 & 24 & 0.66 \\
\hline Sclerolobium melinonii harms & ucshaquiro white & 21.61 & 55 & 0.39 \\
\hline Bauhinia guianensis aublet & ox leg & 7.72 & twenty & 0.39 \\
\hline Apuleia molaris Spruce ex Bentham & caspi cabbage & 20.23 & 37 & 0.55 \\
\hline Cassia reticulata Wilid. & Broom & 69.79 & 102 & 0.68 \\
\hline Balizia pedicellaris(DC.) Barneby \& JW Grimes & pashaco vilco & 32.2 & 80 & 0.40 \\
\hline Erythrina ulei harms & Amasisa & 15.55 & 39 & 0.40 \\
\hline Amazon Aniba Meiz & Moena & 22.7 & 43 & 0.53 \\
\hline Aniba Cinnamon (HBK) Mez. & cinnamon moena & 12.7 & 29 & 0.44 \\
\hline Aniba perutilis Hemsley Kew & black moena & 9.57 & twenty & 0.48 \\
\hline persea mollis (Kunth) Spreng. & moena avocado & $\begin{array}{c}\text { fiftee } \\
n\end{array}$ & 31 & 0.48 \\
\hline Mezilauros synandra (Mez)Kosterm. & Moena & 19 & 39 & 0.49 \\
\hline Nectandra capanahuensis O. Schmidt & Yellow Moena & 5.72 & 10 & 0.57 \\
\hline american persea Mill & Avocado & 4.4 & 10 & 0.44 \\
\hline Eschweilera juruensisKnuth & machimango & 16.26 & 24 & 0.68 \\
\hline Quararibea ochrocalyx (Schumann) Vischer & sapote & 9.42 & 22 & 0.43 \\
\hline Theobroma subincanum C.Martius & peanut & 11.41 & 23 & 0.50 \\
\hline Myconia minutifolia frozen & piece of paper & 13 & $\begin{array}{l}\text { twenty- } \\
\text { one }\end{array}$ & 0.62 \\
\hline Miconia rimachii Wurdack & Myconia & 21.2 & $\begin{array}{l}\text { Four. } \\
\text { Five }\end{array}$ & 0.47 \\
\hline Kunthian guarea A. Juss. & height requirement & 14 & 23 & 0.61 \\
\hline
\end{tabular}


Cedrela fissilisVell.

Cedrela lilloiC.DC.

Brosimum alicastrum Swartz

Ficus guianesis Dev.

ficus insipida willd

Pseudo media laevis (Ruiz \& Pavon)

Claricia racemosa $\mathrm{R}$. and $\mathrm{P}$.

Brosimum acutifolium Hubert.

Maclura tinctoria(L.) D.Don ex Steud. obovate ferrule ducke

Iryanthera juruensisWarburg Virola sebifera aublet

Podocarpus oleifolius D.Don

Cinchona pubescens Vahl

Cinchona delessertiana Standl.

Cinchona micrantha Wow.

Zanthoxylum riedelianum Engler

Manilkara bidentata (ADC) Chev.

Heliocarpus americanus $\mathrm{L}$.

bacciferous urethra L. Gaudich

Cecropia sciadophylla Loefl.

Verbena littoralisHBK

Vitex psudolea Rusby

Vochysia vismiifolia Spruce ex Warming

$\begin{array}{cccc}\text { huascan creed } & 10.41 & 22 & 0.47 \\ \text { lilac cedar } & 8.14 & 19 & 0.43 \\ \text { Manchinga } & 28.2 & \text { Four. } & 0.63 \\ \text { Renaco } & 12.4 & \text { Five } & 0.44 \\ \text { hehe } & 11.1 & 28 & 0.40 \\ \text { chimicua } & 27.98 & 112 & 0.25 \\ \text { Tulpay } & 61.38 & 113 & 0.54 \\ \text { peruvian palo } & 14.96 & 57 & 0.26 \\ \text { Insira } & 19.95 & 29 & 0.69 \\ \text { red cumala } & 32.66 & \text { fifty } & 0.65 \\ \text { red cumala } & 29.51 & 52 & 0.57 \\ \text { white cumala } & 3.5 & 8 & 0.44 \\ \text { strong devil } & 14.81 & 35 & 0.42 \\ \text { yellow cinchona } & 10.9 & 14 & 0.78 \\ \text { Cinchona } & 12.7 & \text { twenty } & 0.64 \\ \text { Quina } & 4.48 & 7 & 0.64 \\ \text { Hualaja } & 8.49 & \text { fifteen } & 0.57 \\ \text { Balata } & 15.93 & 37 & 0.43 \\ \text { Huampo } & 24.4 & 3.4 & 0.72 \\ \text { ishanga } & 4.9 & 16 & 0.39 \\ \text { cecropia } & 50.93 & 125 & 0.41 \\ \text { Verbena } & 6.61 & 14 & 0.47 \\ \text { pali dog } & 32.15 & 56 & 0.57 \\ \text { Cedrillo } & 7.71 & 13 & 0.59\end{array}$

* W: Weight $(\mathrm{g})$

$* \mathrm{~V}$ : Volume $(\mathrm{cm} 3)$

* $\delta$ : Basic density $(\mathrm{g} / \mathrm{cm} 3)$

Table 05.

Total aerial biomass by category and plot $(\mathrm{Kg})$

\begin{tabular}{cccccccc}
\hline \multicolumn{7}{c}{ Total biomass $(\mathbf{K g})$} \\
\hline Category & P1 & P2 & P3 & P4 & P5 & P6 & P7 \\
Low slats (100m2) & 12.69 & 16.45 & 101.35 & 14.61 & 21.08 & 21.30 & 14.94 \\
High slats (625 m2) & 266.37 & 370.52 & 896.44 & 150.77 & 670.68 & 422.19 & 175.57 \\
Stems (2500 m2) & 4473.34 & 9420.13 & 29206.93 & 7175.95 & 30575.24 & 13282.73 & 9684.11 \\
Mature trees (2500 & 6439.25 & 6845.29 & 2111.24 & 74381.65 & 18722.83 & 8201.01 & 5240.29 \\
m2) & 11190.65 & 16652.39 & 32315.96 & 81722.98 & 49989.83 & 21927.23 & 15114.91 \\
Total & & & & & &
\end{tabular}

Table 04 shows the basic densities for tree species, and Table 05 shows the total sum of aerial biomass for each plot, of which plot 4 has the highest value with $81722.98 \mathrm{Kg}$, followed by plot 5 with $49989.83 \mathrm{Kg}$, then there is plot 3, followed by plot 6 , also plot 2 , penultimate plot 7 with 15
$114.91 \mathrm{Kg}$ and finally plot 1 with $11190.65 \mathrm{Kg}$. These results were used to calculate the carbon stored by category of vegetation type and respective plot.

Table 06.

Carbon stored by category of vegetation and plot.

\begin{tabular}{ccccccccc}
\hline \multicolumn{10}{c}{ Stored carbon (t of C/ha) } \\
\hline Category & $\mathrm{P} 1$ & $\mathrm{P} 2$ & $\mathrm{P} 3$ & $\mathrm{P} 4$ & $\mathrm{P} 5$ & $\mathrm{P} 6$ & $\mathrm{P} 7$ & $\overline{\mathrm{X}}$ \\
low poles & 0.63 & 0.75 & 0.82 & 3.34 & 5.07 & 2.50 & 0.73 & 1.98 \\
high poles & 2.13 & 2.44 & 2.96 & 4.39 & 7.17 & 4.87 & 1.21 & 3.60 \\
stems & 8.95 & 18.84 & 58.41 & 14.35 & 61.15 & 26.57 & 19.37 & 29.66 \\
mature trees & 12.88 & 13.69 & 4.22 & 148.76 & 37.45 & 16.40 & 10.48 & 34.84 \\
Total & 24.59 & 35.72 & 66.42 & 170.85 & 110.83 & 50.34 & 31.79 & 70.08 \\
\hline
\end{tabular}


Revista de Investigación Científica Tayacaja Universidad Nacional Autónoma de Tayacaja Daniel Hernández Morillo, Perú Volumen 4 Número 2 - 2021

ISSNe: 2617-9156

Table 06 shows the stored carbon, with an average of 70.08 $\mathrm{t}$ of $\mathrm{C} / \mathrm{ha}$. According to the category of vegetation type, mature trees store $34.84 \mathrm{t}$ of $\mathrm{C} / \mathrm{ha}$, followed by poles with $29.66 \mathrm{t}$ of $\mathrm{C} / \mathrm{ha}$, tall poles with $3.60 \mathrm{t}$ of $\mathrm{C} / \mathrm{ha}$ and low poles with $1.98 \mathrm{t}$ of $\mathrm{C} / \mathrm{ha}$. he has. We also observed that the category of vegetation type that stores the most carbon in its biomass is mature trees with $50 \%$ of the total, followed by poles with
$42 \%$, tall poles with $5 \%$ and low poles with $3 \%$ for each hectare. within the wild zone of the PNTM.

Determination of the behavior of stored carbon based on altitude and category of vegetation type

Table 07.

Frequency distribution of altitude intervals.

\begin{tabular}{|c|c|c|c|c|c|c|c|}
\hline $\mathrm{K}$ & $\mathrm{Xi}$ & x'i & neither & Neither & hi & hi & $\mathrm{tC} / \mathrm{ha}$ \\
\hline one & {$[822-943]$} & 882.5 & two & two & 0.286 & 0.286 & 28.19 \\
\hline two & $\begin{array}{c}{[944-} \\
1065]\end{array}$ & 1004.5 & two & 4 & 0.286 & 0.571 & 51.07 \\
\hline 3 & $\begin{array}{l}{[1066-} \\
1187]\end{array}$ & 1126.5 & one & 5 & 0.143 & 0.714 & 170.85 \\
\hline 4 & $\begin{array}{l}{[1188-} \\
1309]\end{array}$ & 1248.5 & two & 7 & 0.286 & one & 80.59 \\
\hline \multicolumn{3}{|c|}{ Total } & 7 & \multicolumn{3}{|c|}{ one } & \\
\hline $\begin{array}{l}\text { : Int } \\
\text { i: cla } \\
\text { i: } \mathrm{Ac}\end{array}$ & $\begin{array}{l}1 \text { No. } \\
\text { nark } \\
\text { hulated absol }\end{array}$ & equency & $\begin{array}{l}* \mathrm{X} \\
* \mathrm{n} \\
* \mathrm{~h}\end{array}$ & $\begin{array}{l}\text { le interv } \\
\text { e freque } \\
\text { frequen }\end{array}$ & & & \\
\hline
\end{tabular}

* Hi: Accumulated relative frequency

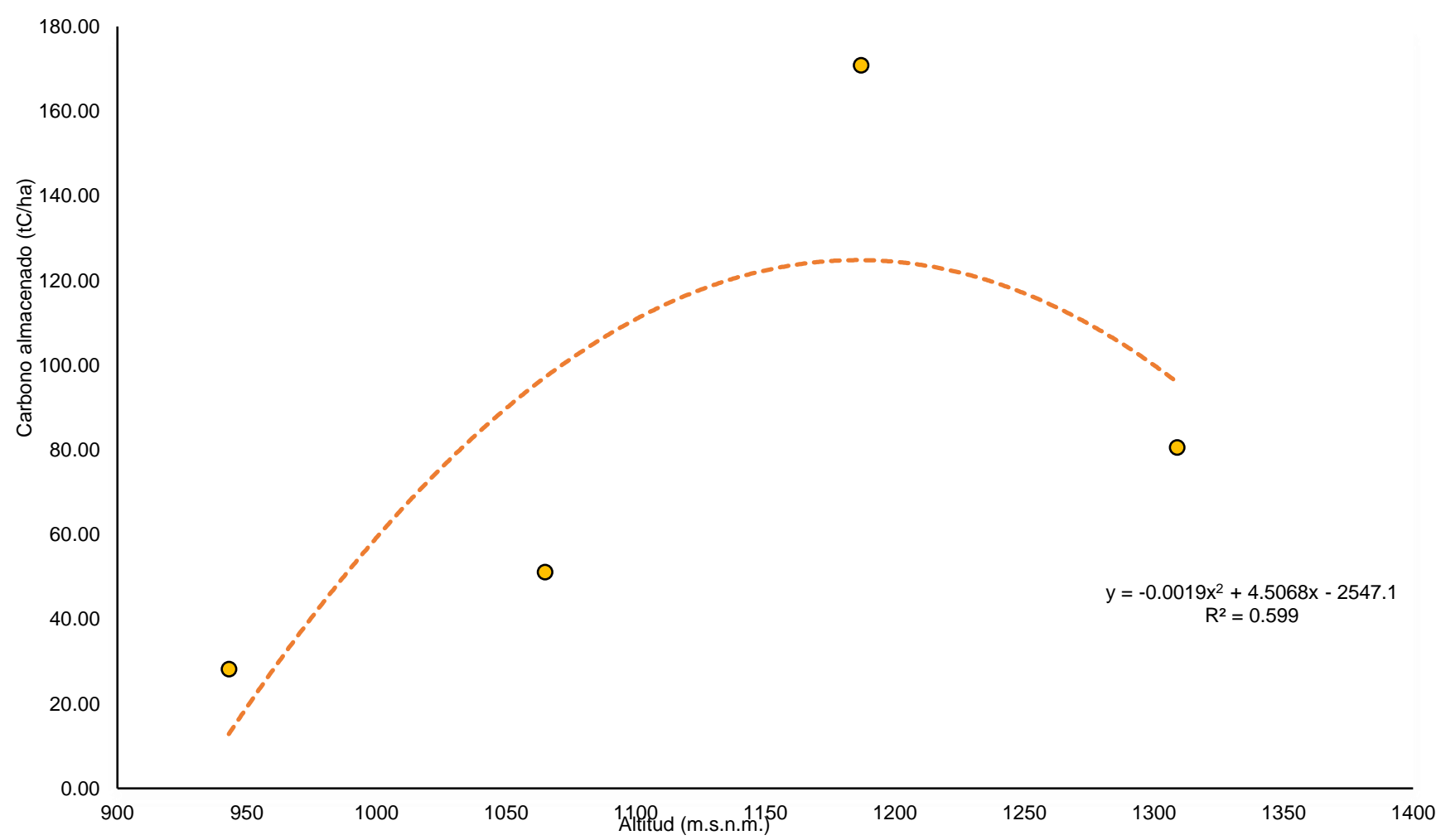

Figure 01.

Behavior of stored carbon with respect to altitude.

Table 07 shows the frequency distribution, which indicates intervals, has its highest peak value in the interval from 1,066 the altitude intervals (masl); In Figure 01, it can be seen that to 1,187 masl. the behavior of the stored carbon with respect to altitude 
Table 08 .

ISSNe: $2617-9156$

Data matrix for statistical analysis.

\begin{tabular}{|c|c|c|c|}
\hline altitude ranges & Category (Type of vegetation) & Carbon (tC/ha) & Carbon stored (tC/ha) \\
\hline \multirow{4}{*}[822-943]{} & low pole & 0.68 & \multirow{5}{*}{28.19} \\
\hline & tall pole & 1.67 & \\
\hline & stems & 14.16 & \\
\hline & mature trees & 11.68 & \\
\hline \multirow{4}{*}[944-1065]{} & low pole & 0.79 & \\
\hline & tall pole & 2.70 & \multirow{3}{*}{51.07} \\
\hline & stems & 38.63 & \\
\hline & mature trees & 8.96 & \\
\hline \multirow{4}{*}[1066-1187]{} & low pole & 3.34 & \multirow{4}{*}{170.85} \\
\hline & tall pole & 4.39 & \\
\hline & stems & 14.35 & \\
\hline & mature trees & 148.76 & \\
\hline \multirow{4}{*}[1188-1309]{} & low pole & 3.79 & \multirow{4}{*}{80.59} \\
\hline & tall pole & 6.02 & \\
\hline & stems & 43.86 & \\
\hline & mature trees & 26.92 & \\
\hline
\end{tabular}

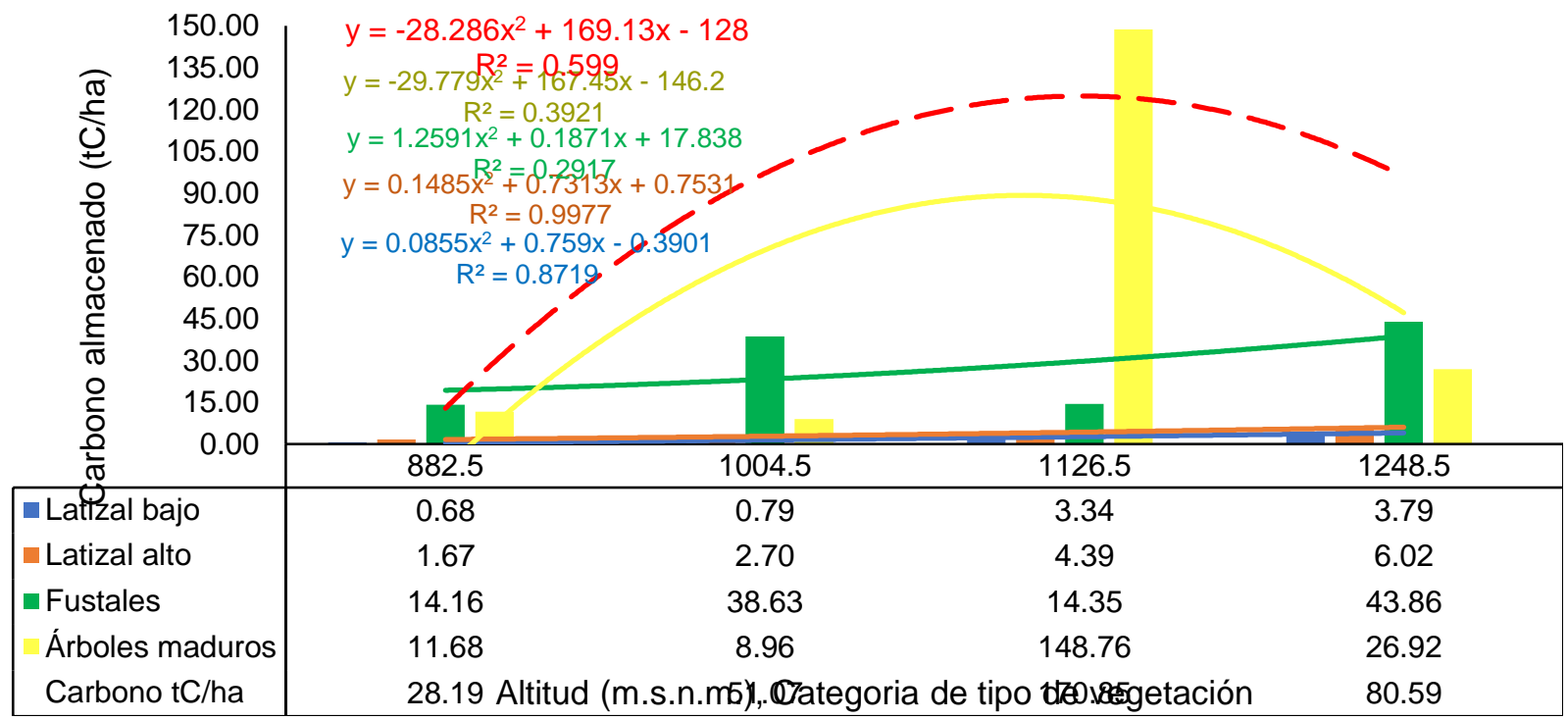

Figure 02.

Carbon behavior according to altitude intervals and vegetation type category.

Table 08 shows the matrix of tabulated data, which served as a basis to be able to work in the IBM SPSS Statistics Trial (Classic student) software. Figure 02 shows us the behavior of the altitude range and the categories of vegetation type with respect to stored carbon. It can be seen that the lines most similar to carbon are defined by mature trees and stems, and a slight influence by the high and low latizales.

Table 09.

ANVA of interaction of altitude, category of vegetation type vs stored carbon.

\begin{tabular}{|c|c|c|c|c|c|}
\hline \multicolumn{6}{|c|}{ ANVA } \\
\hline Variation Source & GL & $\mathrm{SC}$ & $\mathrm{CM}$ & $\mathrm{F}$ & S.I.G.* \\
\hline Altitude & 3 & 5844.58 & 1948.19 & 18.19 & $0.00 *$ \\
\hline Category & 3 & 11872.77 & 3957.59 & 36.96 & $0.00 *$ \\
\hline Altitude*Category & 9 & 22541.47 & 2504.60 & 23.39 & $0.00 *$ \\
\hline Error & 16 & 1713.04 & 107.06 & & \\
\hline Total & 31 & 41971.87 & & & \\
\hline
\end{tabular}

*: Significant

**: Not significant 
In Table 09, it indicates that at a significance level of $95 \%$ the amount of stored carbon is statistically influenced by altitude and vegetation type categories, and the interaction of these two variables evaluated in the field.

\section{Discussion}

Salvador (2014) carried out a study of the diversity of tree species in the Tres de Mayo sector of the PNTM, finding 16 families at different altitude levels, and abundance greater than 131 species with a total sampling of 294 species. Likewise, Valencia (2015) in the study carried out in the Tres de Mayo sector, 28 families and 52 forest species have been found; where at altitudes of 800 to 900 masl the cumala (Virola sebifera Aublet) and shimbillo (lnga altísima Mark) species predominate, from 900 to 1000 masl, the cumala (Virola sebifera Aublet) and cachimbo (Cariniana domestica Ducke) species, from 1000 to 1,100 masl the ishanga species (Urera bacifera L. Gaudich) and black moena (Aniba perutilis Hemsley Kew), from 1,100 to 1,200 masl the chimicua species (Pseudo/media Jaevis (Ruiz \& Pavón)) and shimbillo (lnga altísima Mark), from 1,200 to 1,300 masl the yacushapana species (Terminalia oblonga (R. et. P) Eichler) and shimbillo (lnga altísima Mark), from 1,300 to 1,400 masl the guayabilla species (Rhigospira quadrangularis (Muell. Arg.) Miers) and cumala ( Virola sebifera Aublet), and at altitudes of 1,400 to 1,500 masl the species guayabilla (Rhigospira quadrangularis (Muell. Arg.) Miers) and shimbillo (lnga altísima Mark); In contrast to the results obtained on absolute abundance, we identified 28 families, 73 tree species out of a total of 1837 individuals; the absence of some and the presence of other tree species is due to spatial distribution (physiography), species dispersion and site quality, among other factors. from 1,300 to 1,400 masl the guayabilla (Rhigospira quadrangularis (Muell. Arg.) Miers) and cumala (Virola sebifera Aublet) species, and at altitudes from 1,400 to 1,500 masl the guayabilla (Rhigospira quadrangularis (Muell. Arg.) Miers) and shimbillo species (lnga towering Mark); In contrast to the results obtained on absolute abundance, we identified 28 families, 73 tree species out of a total of 1837 individuals; the absence of some and the presence of other tree species is due to spatial distribution (physiography), species dispersion and site quality, among other factors. from 1,300 to 1,400 masl the guayabilla (Rhigospira quadrangularis (Muell. Arg.) Miers) and cumala (Virola sebifera Aublet) species, and at altitudes from 1,400 to 1,500 masl the guayabilla (Rhigospira quadrangularis (Muell. Arg.) Miers) and shimbillo species (lnga towering Mark); In contrast to the results obtained on absolute abundance, we identified 28 families, 73 tree species out of a total of 1837 individuals; the absence of some and the presence of other tree species is due to spatial distribution (physiography), species dispersion and site quality, among other factors. In contrast to the results obtained on absolute abundance, we identified 28 families, 73 tree species out of a total of 1837 individuals; the absence of some and the presence of other tree species is due to spatial distribution (physiography), species dispersion and site quality, among other factors. In contrast to the results obtained on absolute abundance, we identified 28 families, 73 tree species out of a total of 1837 individuals; the absence of some and the presence of other tree species is due to spatial distribution (physiography), species dispersion and site quality, among other factors.

Sosa (2016) states that forest biomass is currently an important element in studies on the changes that occur on a global scale, thanks to the attenuating effect (sink) that forests and related systems can have by sequestering excess greenhouse gases. greenhouse, temporarily (biomass) and permanently (soil). In the case of Tingo María National Park, it is an area protected by the state, which already plays an important role in the face of global problems and reduces and stores a large proportion of $\mathrm{CO} 2$ from the atmosphere. Manta (1988) indicates that a forest is out of risk and management can be carried out if there are at least 150 individuals per hectare with a DBH between 10 and $40 \mathrm{~cm}$, a category corresponding to stems, therefore.

Güere (2015) in the study carried out in the Tingo María National Park, indicates that the stored carbon found is influenced by $56.73 \%$ specifically by mature trees with more than $40 \mathrm{~cm}$ in diameter at breast height, followed by $29.9 \%$ in the stems. , joining both categories exceed $3 / 4$ parts of the total stored carbon found; the results obtained in the present investigation indicate that mature trees store $50 \%$ of the carbon per hectare, followed by poles with $42 \%$, tall poles with $5 \%$ and low poles with $3 \%$; These results ratify what was obtained in the 2015 study, the influence obtained was $92 \%$ for the wild area of the PNTM. According to Güere (2015) they state that forests that have net growth are capable of net absorption and therefore contribute to the reduction of atmospheric $\mathrm{CO} 2$; due to the little or no alteration in its habitat, such as the wild area, since it is protected and little altered.

IDEAM (2010) indicates that the content of biomass and carbon potentially stored in the forests of the National Natural Parks system of Colombia, specifically for tropical humid forests, the average carbon value for this type of natural forest is $130.44 \mathrm{tC} / \mathrm{ha}$, compared with the results obtained of 70.08 $\mathrm{tC} / \mathrm{ha}$ can be considered representative, if it is taken into account that the study only worked on the tree aerial biomass, and not the other carbon storage components of an area. It is important to bear in mind that, due to the significant carbon content of these primary forests, they also have a high potential to become sources of $\mathrm{CO} 2$ emissions; therefore, surveillance control must be taken into account so that the surrounding population does not affect the natural forest.

Gonzales (2013) mentions that criteria such as type of forest or vegetation, density of wood, adjustment factors based on biomass data calculated from volumes per hectare of forest inventories, as well as conditions are taken into account. of the site, such as location and climate. Cubero and Rojas (1999), point out that the carbon content in tree biomass is influenced by the quality of the site and age of the plantations, the results obtained indicate that mature trees (older) and stems are the ones that stored the greatest the carbon in the biomass, and also to other factors mentioned by previous authors that are the density, years of life, altitude, 
ISSNe: 2617-9156

climate conditions and quality of the site where they develop. Cuellar and Salazar (2016) in a study carried out on the dynamics of stored carbon indicate that the warm and rainy climate of humid tropical forests generates rapid plant growth and most of the carbon is found in the vegetation. Carbon stocks, as in the case of the PNTM with a humid tropical forest, vary considerably depending on the abundance of trees and factors that affect their growth.

\section{Conclusions}

The carbon in the living tree aerial biomass of the wild area of the Tingo María National Park was on average $70.08 \mathrm{tC} / \mathrm{ha}$.

The carbon in the living tree aerial biomass of the wild area of the Tingo María National Park, according to the categories of vegetation type, was $50 \%(49.08 \mathrm{tC} / \mathrm{ha})$ for mature trees, $42 \%(27.75 \mathrm{tC} / \mathrm{ha})$ for poles, $5 \%(3.82 \mathrm{tC} / \mathrm{ha})$ for tall poles and $3 \%(2.15 \mathrm{tC} / \mathrm{ha})$ for low poles.

\section{REFERENCES}

[1] Allen, W. (2001). Statistics applied to business and economy. Trad. by Yelka Garcia. 3ed. Bogota, Colombia, McGraw-Hill. 543p.

[2] Borrero, J. (2012). Aerial biomass and carbon content in the campus of the Pontificia Universidad Javeriana in Bogotá. Thesis for Ecologist Degree. Bogota Colombia. Pontifical Javeriana University. 54p.

[3] Chave J, Andalo S, Brown A, Cairns J, Chambers H, Folster F, Fromard N, Higuchi T, Kira J, Lescure B, Nelson H, Ogawa H, Puig B, Riera Y, Yamakura. (2005). Tree allometry and improved estimation of carbon stocks and balance in tropical forests. Oecology 145: 87-99

[4] Cubero, JY, Rojas, R. (1999). Carbon fixation in plantations of Gmelina arborea, Tectona grandis and Bombacopsis quinata in the cantons of Hojancha and Nicoya. Thesis (Bachelor's Degree in Forest Sciences). National University. Guanacaste, Costa Rica. 93 pages

[5] Cuellar, J., Salazar, E. (2016). Dynamics of carbon stored in the different systems of land use in Peru. Lima Peru. National Institute of Agrarian Innovation INIA. 217 pages

[6] Dominguez, E. (2010). Instruments for measuring dasometric variables. Foundations and elaboration with the student of the training cycle "Higher Technician in Management and Organization of Landscape Natural Resources". Innovation and Educational Experiences. Innovation and Educational Experiences. Cordoba, Argentina, 9 p.m.

[7] Gonzales, P. (2013). Economic assessment of CO2 sequestration in plantations of Vochysia lomatophylla (standl) "quillosisa" of different ages at CIEFOR Puerto Almendra. Thesis (Tropical Forest Ecology Engineer). National University of the Peruvian Amazon. Faculty of Forest Sciences. Iquitos-Peru. 74 p.

[8] Guere, F. (2015). Carbon stored in the protected forest area of the Tingo María National Park. Thesis Mg. Sc. In Agroecology Mention in Environmental Management. Tingo Maria, Peru. Postgraduate School of the National Agrarian University of La Selva. 109 pages

[9] IDEAM. (2010). Executive summary of the technical report on the estimation of the potential reserves of carbon stored in the aerial biomass in the natural forests of Colombia, Institute of Hydrology, Meteorology and Environmental Studies of Colombia. Bogota DC (Colombia). 42p.

[10] IPCC. (2001). The Carbon Sinks. Retrieved from www.cescyl.es/pdf/coleccionestudios/Pkioto.pdf.

[11] IPCC. (2003). Integrated pollution control and prevention. Retrieved from http://eippcb.jrc.ec.europa.eu/reference/BREF/lvo_bref _0203.pdf.

[12] IPCC. (2018). Impacts of $1.5^{\circ} \mathrm{C}$ Global warming on natural and human systems. IPCC special report. Recovered fromhttps://www.ipcc.ch/site/assets/uploads /sites/2/2019/05/SR15_SPM_version_report_LR.pdf.

[13] Lamprecht, H. (1990). Forestry in the Tropics: Forest ecosystems in tropical forests and their tree species; possibilities and methods for sustained use. (Deutsche Gesellschaft für Technische Zusammenarbeit) GTZ. German Federal Republic, Germany. 335p.

[14] Blanket, M. (1988). Silvicultural analysis of two types of humid lowland forest on the Atlantic slope of Costa Rica. Thesis. mg. SC. Turrialba, Costa Rica. CATIE. 137 pages

[15] Morels, A. (2007). Sampling in finite populations. Retrieved from http://webpersonal.uma.es/ morillas/muestreo.pdf.

[16] Murray, R., Jacobson, M. (1982). An evaluation of dimension analysis for predicting shrub biomass. Journal of Range Management 35:451-454.

[17] Ortiz, M. (1993). Techniques for estimating the growth and yield of individual trees and forests. Carthage, Costa Rica. Technological Institute of Costa Rica. 71 p.

[18] Pinelo, G. (2000). Manual for the establishment of permanent sampling plots in the Maya Biosphere 
Reserve, Peten, Guatemala. Turrialba, Costa Rica. Tropical Agronomic Center for Research and Teaching. 68 .

[19] Salvador, A. (2014). Diversity of tree species (decits/individual) as an indicator of environmental quality at the different levels of the Tingo María National Park. Pre-professional practice. Tingo Maria, Peru. Academic Department of Environmental Sciences. 71p.

[20] Sosa, J. (2016). Economic valuation of CO2 sequestration in three types of forest in the District of Alto Nanay, Loreto - Peru - 2014. Thesis for Forestry Engineer. Iquitos, Peru. School of Forestry Engineering of the National University of the Peruvian Amazon. 99p.

[21] Valencia, R. (2015). Diversity of forest species in the wild area of the Tres de Mayo sector of the Tingo María National Park. Thesis for RNR Forestry Mention Engineer. Tingo Maria, Peru. Faculty of RNR of the National Agrarian University of La Selva. 140p.

[22] Vallejo, M., Londoño A., López R., Galeano G., Álvarez E., Devia W. (2005). Establishment of permanent plots in forests of Colombia. Bogota DC, Colombia. Alexander von Humboldt Biological Resources Research Institute. 310 p.

[23] Vargas, V. (2007). Descriptive Statistics for Environmental Engineering with SPSS. Cali, Colombia. FERIVA SA 312 p printer.

[24] Wieman, M., Williamson, G. (1989). Wood specific gravity gradients in tropical dry and montane rain forest trees, American Journal of Botany 76 (6): 924-928. 